\title{
Aqueous-Organic Phase Transfer of Gold Nanoparticles and Nanorods using an Ionic Liquid \\ Guor-Tzo Wei ${ }^{*}$ Z Zusing Yang, Chia-Yin Lee, Hsiao-Yen Yang and C. R. Chris Wang
}

\section{Supporting Information}

\section{Experimental Procedures and Analysis of Gold Nanoparticles and Nanorods.}

UV-Vis Absorption Spectrometry. UV-Vis absorption spectra of gold nanoparticles and nanorods were obtained using a double-beam UV-Vis-NIR spectrometer (Shimadzu UV$3100 \mathrm{PC})$. The spectra of the gold nanoparticles and nanorods in $\left[\mathrm{C}_{4} \mathrm{MIM}\right]\left[\mathrm{PF}_{6}\right]$ have been background corrected to take into account the presence of the ionic liquid.

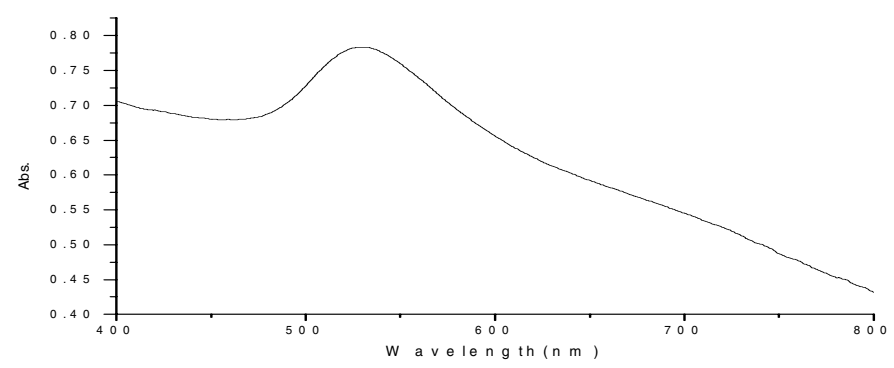

Figure S1. A typical UV-Vis spectrum of gold nanoparticles in $\left[\mathrm{C}_{4} \mathrm{MIM}\right]\left[\mathrm{PF}_{6}\right]$.

Phase Transfer of Gold Nanoparticles with Different Shapes. An aqueous solution containing spherical and rod shapes of gold nanoparticles was added to the $\left[\mathrm{C}_{4} \mathrm{MIM}\right]\left[\mathrm{PF}_{6}\right]$ at the volume ratio of 3 to 1 . Figure S2 presents UV-Vis spectra recorded the aqueous solution before and after phase transfer. Photographs of the test tubes containing the biphasic aqueous/IL mixtures are displayed in the inset of Figure S2. The plasmon resonance peak of the gold nanorod in the aqueous layer (curve a) disappeared upon phase transfer (curve b). 


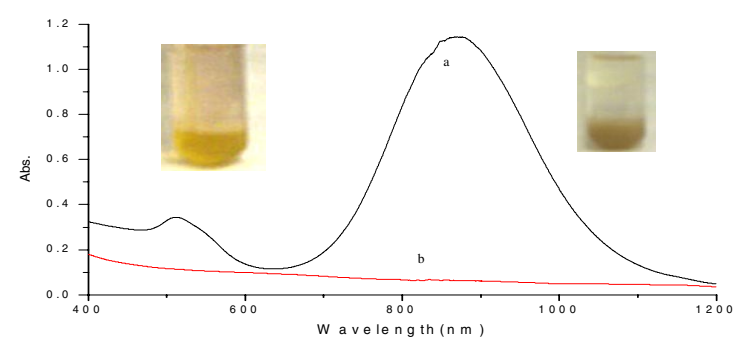

Figure S2. UV-Vis spectra of the aqueous layer of the solution of gold nanorods (a) before and (b) after phase transfer with $\left[\mathrm{C}_{4} \mathrm{MIM}\right]\left[\mathrm{PF}_{6}\right]$. The insets display test tubes containing gold nanoparticles before (left) and after (right) phase transfer with the IL

Transmission Electron Microscopy. Each aqueous sample was placed on a carbon-coated grid, the grid was covered, and the solution was allowed to dry. For nanoparticles and nanorods in $\left[\mathrm{C}_{4} \mathrm{MIM}\right]\left[\mathrm{PF}_{6}\right]$, the excess ionic liquid and surfactant on the grid were removed from the grid by rinsing carefully with ethanol. The nanoparticles were examined using a Hitachi h-700h transmission electroscope and the size distribution of the nanoparticles was estimated using Sigma Scan software.
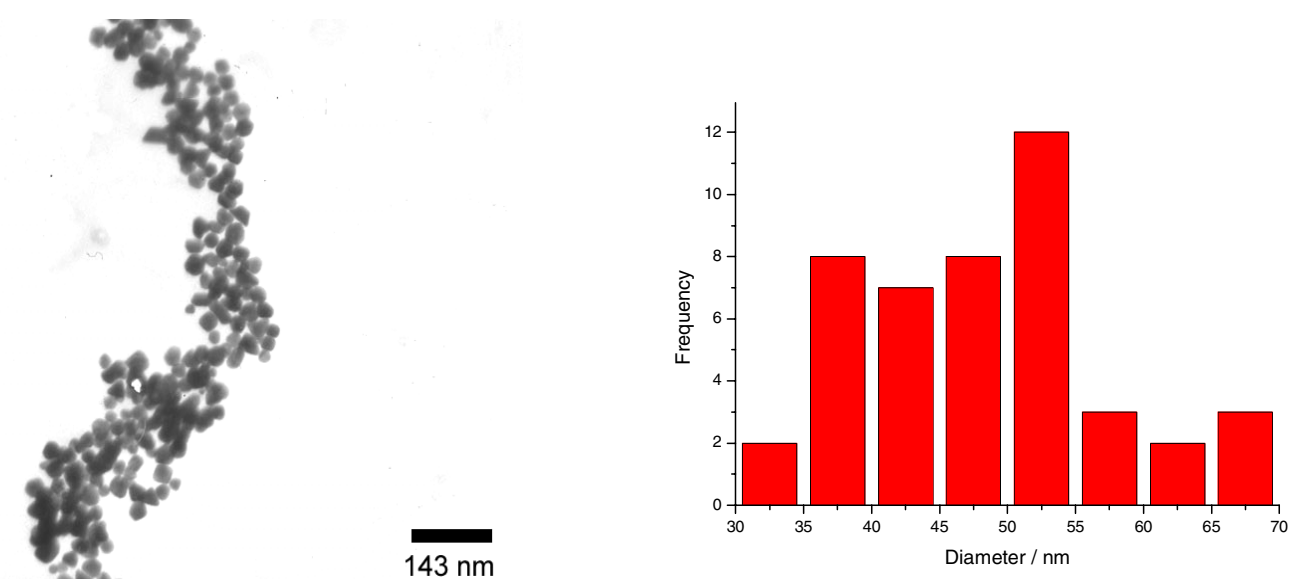

Figure S3. TEM image (left) and histogram (right) displaying the distribution of sizes for the Au nanoparticles in the aqueous phase. The average diameter is ca. $48.1 \pm 9.0 \mathrm{~nm}$. 

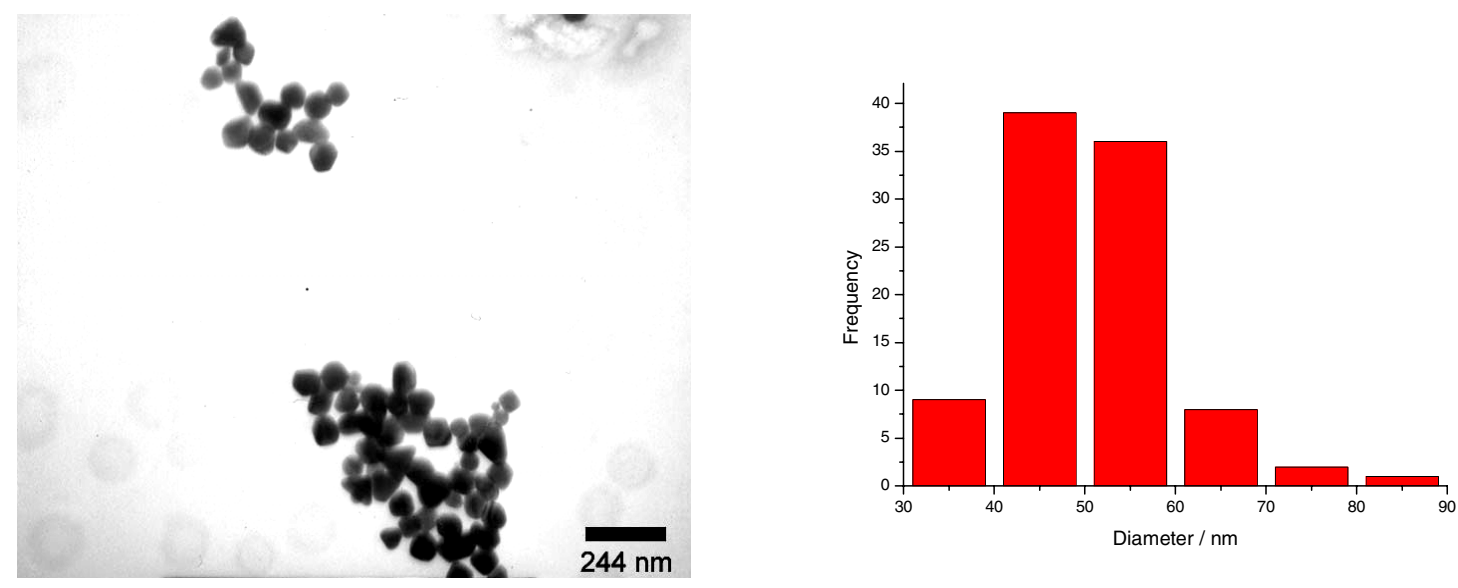

Figure S4. TEM image (left) and histogram (right) displaying the distribution of sizes for the Au nanoparticles in $\left[\mathrm{C}_{4} \mathrm{MIM}\right]\left[\mathrm{PF}_{6}\right]$. (average diameter $=49.9 \pm 9.3 \mathrm{~nm}$ ).
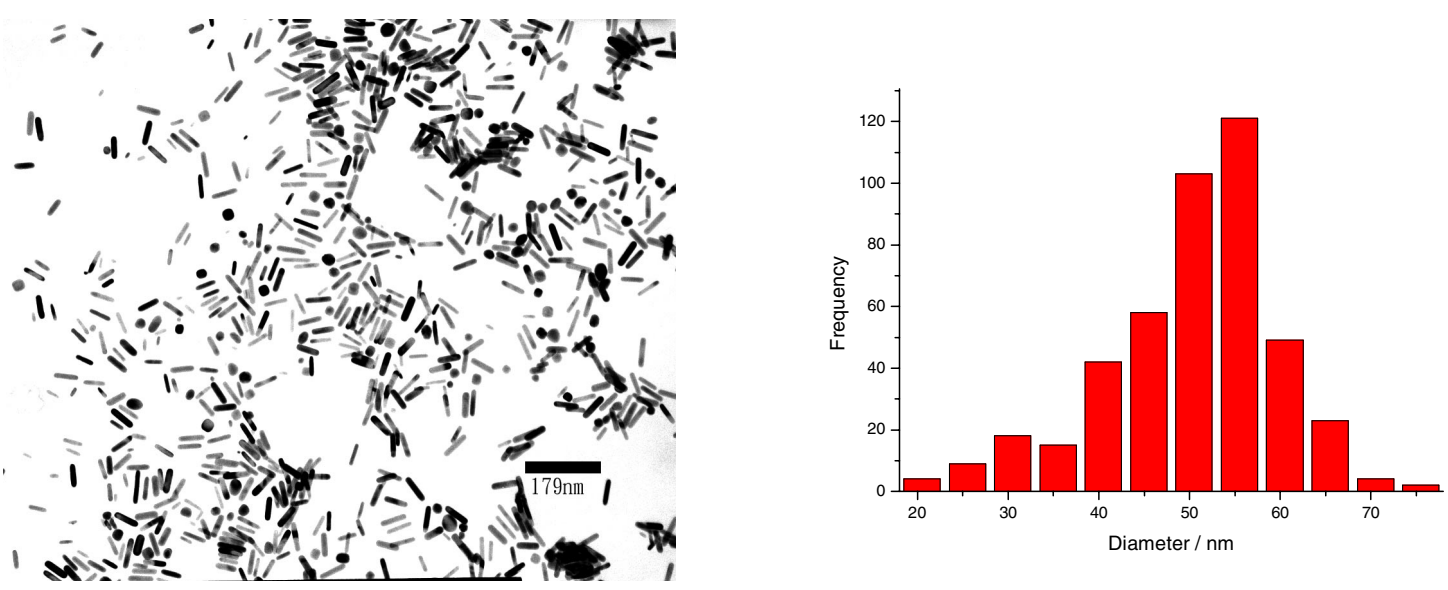

Figure S5. TEM image (left) and histogram (right) displaying the distribution of sizes for the Au nanorods in the aqueous phase. The average length of the rods is $50.4 \pm 9.5 \mathrm{~nm}$.
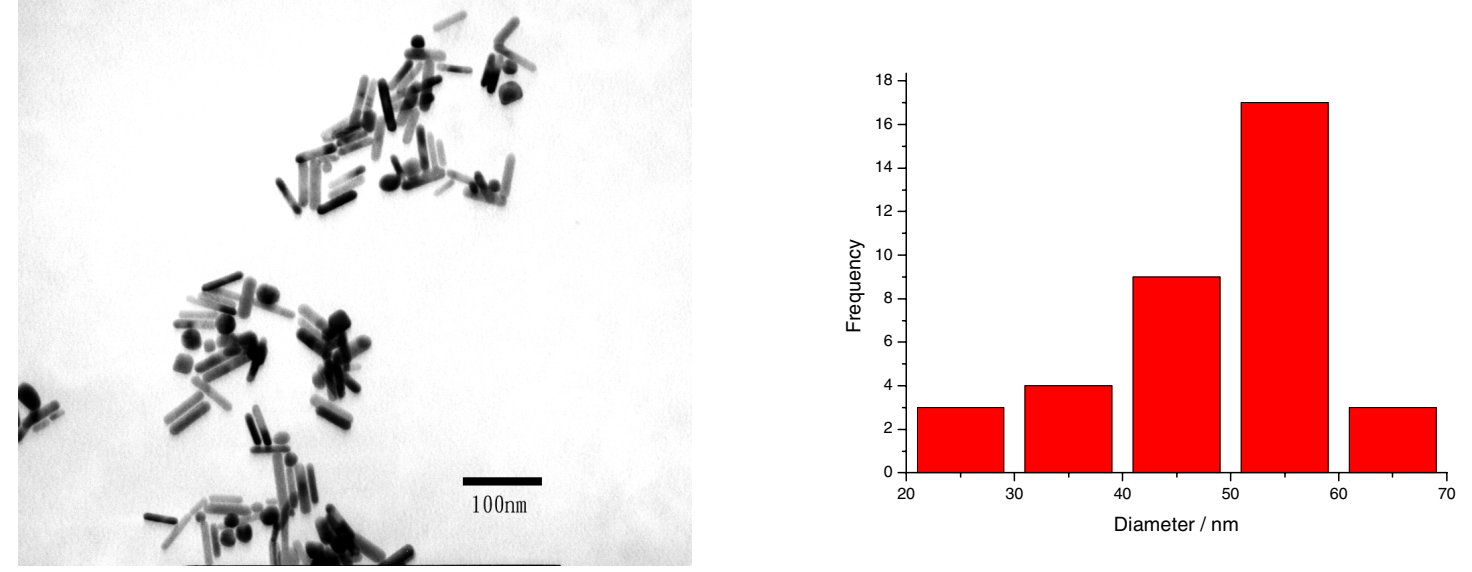
Figure S6. TEM image (left) and histogram (right) displaying the distribution of sizes for the Au nanorods in $\left[\mathrm{C}_{4} \mathrm{MIM}\right]\left[\mathrm{PF}_{6}\right]$. The average length of the rods is $47.6 \pm 11.2 \mathrm{~nm}$.

The Effect of Salt Concentration on the Phase Transfer of Gold Nanoparticles. To examine the effect of salt concentration on the phase transfer process using polar and nonpolar solvents, we added different concentrations of $\mathrm{N}$-tetrabutylammonium bromide (TBABr) to the organic phase. TBABr was employed to mimic the role of the salt in the organic phase and for its solubility in organic solvents. The absorption of the gold nanoparticles in the aqueous phase was measured after phase transfer with the organic solvents in which different concentrations of the salt had been added.

Table S1. The effect of salt concentration on the aqueous-organic phase transfer of gold nanoparticles using (a) a polar and (b) a nonpolar solvent.

(a) 10-Undecen-1-ol.

\begin{tabular}{|l|l|}
\hline$[\mathrm{TBABr}]$ added & Extraction efficiency increased (\%) \\
\hline $0 \mathrm{ppm}$ & \\
\hline $1000 \mathrm{ppm}$ & 4.88 \\
\hline $3000 \mathrm{ppm}$ & 11.4 \\
\hline $5000 \mathrm{ppm}$ & 19.0 \\
\hline $6000 \mathrm{ppm}$ & 21.6 \\
\hline
\end{tabular}

(b) Benzene

\begin{tabular}{|l|l|}
\hline$[\mathrm{TBABr}]$ added & Extraction efficiency increased (\%) \\
\hline $0 \mathrm{ppm}$ & \\
\hline $2000 \mathrm{ppm}$ & 1.72 \\
\hline $4000 \mathrm{ppm}$ & 5.07 \\
\hline $6000 \mathrm{ppm}$ & 5.33 \\
\hline $8000 \mathrm{ppm}$ & 6.74 \\
\hline
\end{tabular}

The Effect of Salt Concentration on the Phase Transfer of Gold Nanoparticles. To examine the effect of the hydrophobic tail of the salt on the efficiency of the phase transfer process, we replaced TBABr, employed above, with TTAB and employed it in the phase transfer using traditional organic solvents. The absorption of the gold nanoparticles in the aqueous medium after phase transfer was measured to estimate the efficiency of phase transfer, as indicated in Table S2. 
Table S2. The effect of the concentration of TTAB on the aqueous-organic phase transfer of gold nanoparticles using nonpolar solvents.

\begin{tabular}{llll}
\multirow{2}{*}{$\begin{array}{l}\text { Conc. of TTAB } \\
\text { Added }\end{array}$} & \multicolumn{3}{c}{ Extraction efficiency increase (\%) } \\
\cline { 2 - 4 } & Benzene & Hexane & Chloroform \\
\hline $30 \mathrm{mM}$ & 0 & 0.96 & 1.02 \\
$60 \mathrm{mM}$ & 1.59 & 0.96 & 2.17 \\
$90 \mathrm{mM}$ & 3.06 & 1.10 & 3.00
\end{tabular}

